\title{
Band Offset Engineering in C-functionalized Boronitrene
}

\author{
K. O. Obodo ${ }^{1,2},{ }^{*}$ N. Chetty ${ }^{2,3}$, and J. T. Obodo ${ }^{4 \dagger}$ \\ ${ }^{1}$ Physics Department, University of South Africa, \\ Muckleneuk Campus Pretoria 003; ${ }^{2}$ Physics Department, University of Pretoria, \\ Pretoria 0002, South Africa; ${ }^{3}$ National Institute for Theoretical Physics, Johannesburg, 2000, \\ South Africa; and ${ }^{4}$ Department of Physics and Astronomy, University of Nigeria, Nsukka, Nigeria.
}

\begin{abstract}
Using density functional theory within the generalized gradient approximation (GGA), we explore the elastic and electronic properties of monolayer boronitrene containing a double line of substituted carbon atoms. This C-functionalized boronitrene structure, referred to as BN/C zigzag-short, has a significantly reduced band gap of about $1.79 \mathrm{eV}$ due to the presence of carbon in the lattice but shows no significant change in its elastic properties compared to pristine boronitrene. The effect of strain on the band offset is explored for various applied strains. It is shown that the band offset changes significantly as a function of biaxial and uniaxial strain, opening an avenue for band offset engineering.
\end{abstract}

PACS numbers: 61.48.Gh, 68.35.bg, 73.22.-f

\section{INTRODUCTION}

Single layer $h$-BN (2D hexagonal boron nitride preferably called boronitrene) is attracting considerable research and technological attention due to the recent interest in novel monolayer materials. ${ }^{1-9}$ These $2 \mathrm{D}$ materials are becoming instrumental in the development of nanoscale electronic applications. ${ }^{10-12}$ Graphene has led the way with its unique electronic and mechanical properties. Since its mechanical exfoliation from graphite ${ }^{13}$ in 2004, other 2D materials, including $h$-BN, have been cleaved to produce monolayer flakes. ${ }^{14}$ These flakes are stable at room temperature and of very high quality. Recently CVD has been used to grow boronitrene layers ${ }^{15,16}$ thereby opening the way for large-scale industrial applications. Ref. 10 suggest that the possibility of patterning defective boronitrene structures as the one studied can be achieved by using controlled heavy-ion bombardment in a C-rich environment.

Ab initio studies ${ }^{17-19}$ and experimental results ${ }^{13,14}$ indicate that boronitrene is a wide band gap material whereas graphene is a zero band gap semi-metal. This makes boronitrene an ideal complimentary material to graphene for nanoscale electronic applications. The band gap in boronitrene can be easily modified due to the absence of long-range inter-planar interactions. Recently, the authors ${ }^{20}$ investigated the modification of the band offset in a single layer of boronitrene by substituting a double line of carbon atoms. This effectively introduces a line of dipoles at the interface, which results in a step in the potential across the barrier resulting in a band offset. The zigzag-short orientation is energetically the most favorable configuration among other configuration considered in our previous study, with a heat of formation of $0.502 \mathrm{eV}$ and a band offset of $1.51 \mathrm{eV}$ when compared with the zigzag-long structure, which has a heat of formation of $1.764 \mathrm{eV}$ and a band offset of $1.99 \mathrm{eV}$. In this paper, we shall refer to this structure simply as the "zigzag short" structure. This structure is constructed from boronitrene by replacing the $\mathrm{B}$ and $\mathrm{N}$ atoms along a single chain in the zigzag direction by $\mathrm{C}$ atoms. The two lines of $\mathrm{C}$ atoms parallel to the interface are a "short" distance apart compared with the other configurations that were considered in that work.

Band gap engineering is a subject of much investigation in $2 \mathrm{D}$ and $3 \mathrm{D}$ systems due to its potential in device applications ${ }^{11,21-23}$. Li et al. ${ }^{24}$ studied the effect of various strain configurations on the electronic properties of pristine boronitrene. They demonstrated that the band gap can be indirect or direct depending on the applied strain and they showed that strain significantly reduces the band gap. This provides an avenue for switching boronitrene from an insulator to a semi-conductor under lateral structural deformations.

Graphene and boronitrene are isostructural with a slight lattice mismatch; their elastic properties show no pronounced difference. Recently, the elastic constants for both graphene and boronitrene were calculated using DFT, and the various elastic moduli for these two materials were compared. ${ }^{25}$ It was found that the measure of the resilience to stretching (the layer modulus $\gamma$ ) for boronitrene has a value $\sim 85 \%$ that of graphene with graphene having a value of $206 \mathrm{~N} \mathrm{~m}^{-1}$. The $2 \mathrm{D}$ Young's modulus has a value $\sim 81 \%$ that of graphene at $275.8 \mathrm{~N} \mathrm{~m}^{-1}$. This is in accordance with a theoretical survey of monolayer group-IV elements and III-V binary honeycomb compounds $^{18}$ that predicted that graphene has the highest in-plane Young's modulus with boronitrene being placed second. These studies show that although boronitrene is the weaker of the two, its elastic strength is comparable to that of graphene giving it great structural strength and resilience. Also, using molecular mechanical simulations, the effect of vacancies was shown to reduce the in-plane Young's modulus for boronitrene significantly for concentrations below $10 \% .{ }^{16}$ It remains to be determined how the substitutions of atoms in the zigzag-short structure alter the elastic properties of boronitrene. It is also unknown how this modified structure compares elastically to the pristine boronitrene. Along these lines, the elastic properties of "zigzag-short" boronitrene is investigated 
in the current study.

In this paper we investigate the effects of externally applied strain on the band offset of the $\mathrm{BN} / \mathrm{C}$ zigzag-short structure. We consider the effects of various different orientations of the applied strain, namely uniaxial and biaxial strain. This consequently affects its electronic and structural properties which we investigate.

We give a brief description of the computational methods that is used to analyse the changes to the electronic and structural properties of $\mathrm{BN} / \mathrm{C}$ zigzag-short in section II. In section III, we discuss the details of the BN/C zigzag-short structure and the effect of the various strains on the electronic band structure and band offset of this system. The elastic properties and phonon dispersion of $\mathrm{BN} / \mathrm{C}$ zigzag-short structure is presented in section IV. Finally, we summarize our conclusions in section $\mathrm{V}$.

\section{METHODOLOGY}

The calculations for the band structure are performed using density functional theory ${ }^{26}$ as implemented in the VASP $\operatorname{code}^{27}$ using the Projected Augmented Wave formalism $^{28}$ (PAW). We use the PBE exchange-correlation functional $^{29}$ for the generalized gradient approximation (GGA). A kinetic energy cutoff of $550 \mathrm{eV}$ is chosen to ensure adequately converged total energies. For the BN/C zigzag-short structure, 20 atoms per unit cell ( 9 Boron, 9 Nitrogen and 2 Carbon atoms) are used to ensure isolated junction images. The BN/C zigzag-short structure used in this study is a fully converged semi-infinite layer in the $\mathrm{x}$-direction with the junction parallel to the $\mathrm{y}$-axis. The material as mentioned above is a monolayer. To prevent interactions between its periodic images in the out-ofplane direction, an optimized vacuum distance of 15 is found to be adequate.

The elastic constants are calculated using the leastsquares fit method ${ }^{33}$ as implemented in the MedeA-MT module. Here also, the tetrahedron method is used for the Brillouin zone integrations. The elastic moduli are calculated from the planar elastic constants as well as the longitudinal, shear and mean acoustic velocities and the 2D Debye temperatures. Moduli for these 2D structures are in units of $\mathrm{Nm}^{-1}$ unlike the 3D structures, which are in $\mathrm{Nm}^{-2}$. The elastic constants need to be positive to fulfill the criteria of elastic stability.

The expressions presented below are used to evaluate the $2 \mathrm{D}$ shear, longitudinal and mean velocities:

$$
\begin{gathered}
V_{t}=\sqrt{\frac{c_{66}}{\rho}} \\
V_{l}=\sqrt{\frac{c_{11}}{\rho}} \\
V_{m}=\left(\frac{1}{2}\left(\frac{1}{V_{l}^{2}}+\frac{1}{V_{t}^{2}}\right)\right)^{-\frac{1}{2}}
\end{gathered}
$$

where $V_{t}, V_{l}, V_{m},\left\{c_{11}, c_{66}\right\}$ and $\rho$ are the shear velocity, longitudinal velocity, mean velocity, elastic constants and density respectively.

The Debye temperature is evaluated using:

$$
\Theta_{D}=\frac{k_{B} V_{m}}{\hbar} \sqrt{\frac{n 4 \pi}{A}}
$$

where $\Theta_{D}, k_{B}, \hbar, n$ and $A$ are the Debye temperature, Boltzmann constant, Planck's constant, number of ions and the area respectively.

The sum of the Hartree, local ionic and exchangecorrelation potentials are used to calculate the macroscopic potential across the junction. Planar averaging of the charge density and potential are done over the $y z$ planes as a function of $x$, as this is equivalent to line averaging in the plane of the single layered material, where $f(x, y, z=0)$.

We observe the polarity of the zigzag-short structure by considering how the potential varies across the junction. It is useful to consider the potential (represented by $f$ below) averaged along lines parallel to the interface:

$$
\tilde{f}(x)=\frac{1}{L} \int f(x, y) d y
$$

The interface region is unambiguously defined as the region where both the charge density and potential significantly deviate from constancy, whereas the rest are bulk. In order to eliminate the effects from the bulk system, the macroscopic average for the each line-averaged quantity $^{34}$ is calculated to emphasize the behavior at the junction.

$$
\tilde{\tilde{f}}(x)=\frac{1}{a} \int_{x-a / 2}^{x+a / 2} \tilde{f}\left(x^{\prime}\right) d x^{\prime}
$$

Here, the macroscopic average is computed over the periodic length $a$.

The band offset (which is the relative alignment of the energy bands at a semiconducting heterojunction) can be easily determined from the macroscopic average of the potential across the junction as shown in our previous study $^{20}$ using this scheme. The band gap as a function of various strain configurations is not within the scope of the current investigation. However, as mention above $\mathrm{Li}$ et al investigated the band gap versus strain on pristine boronitrene. Noteworthy the BN/C systems at infinitely large distance from the junction, the structures are essentially boronitrene.

The phonon dispersion calculated exploits the linearity relationship between the induced forces on the atoms in the crystal and the displacement of the atoms from their equilibrium position, which holds within the harmonic regime. 


\section{STRUCTURAL AND ELECTRONIC PROPERTIES}

Fig. ?? shows the zigzag-short structure which has the Pmma space group symmetry, and obviously has lower symmetry compared to boronitrene $\left(P-6_{3} m m c\right)$. It possesses an underlying honeycomb structure. We have discussed the zigzag-short structure in detail in our previous study. ${ }^{20}$ This structure resides in the $x y$-plane with the junctions' parallel to the $y$-axis. The lattice constant for the supercell with length $\mathrm{Lx}$, Ly and $\mathrm{Lz}$ are 13.04, 2.51 and $15.00 \AA$ respectively.

Fig. 2 shows the calculated band structures for boronitrene and zigzag-short. A supercell size of 12 atoms for both structures is used for consistency. This is because no significant difference in the band gap or states close to the Fermi level with the larger cell, just more bands in the electronic structure diagram due to the larger number of atoms. Hence for more clarity the smaller unit cell was used. Boronitrene is a wide-band gap semi-conductor with a calculated indirect band gap of about $4.58 \mathrm{eV}$. This can be attributed to the bonding and antibonding combinations of N-pz and B- $p z$ orbitals with N-pz pronounced for the filled band at the edge of valence band. ${ }^{17}$ While, the zigzag-short structure has a reduced band gap of about $1.79 \mathrm{eV}$ with the valence band maximum and conduction band minimum located between $\mathrm{Y}$-T in the Brillouin zone, where $\mathrm{Z}=(0.5,0.5,0.5)$ similar to $(\mathrm{R}$, $\mathrm{L}) ; \mathrm{T}=(0,0.5,0.5) ; \mathrm{Y}=(0,0.5,0)$ and $\Gamma=(0,0$, $0)$. This reduction is attributed to the two additional bands introduced close to the Fermi energy level due to the insertion of the double line of carbon atoms at the interface. The effect of strain on the band structure has not been investigated in the current study for this structure. However, strain would effectively modify the highest occupied molecular molecular orbital and lowest unoccupied molecular orbital leading to the change in the electronic structure. This is evident from the band-offset.

Fig. 1 shows the calculated line and macroscopic averaged potential of the $\mathrm{BN} / \mathrm{C}$ zigzag-short structure. The line-averaged and macroscopic averaged potential are the solid and dotted line respectively. The calculated band offset, given as $\Delta V$, is the difference between the two parallel line drawn across the macroscopic potential. The band offset arises due to the discontinuity of the potential across the interfacial region.

The ground state structures for the various strain configurations were determined by carrying out full structural optimizations in the strained directions. For the relaxed ground state, the interface is composed of a zigzag chain of $\mathrm{C}$ atoms with fully relaxed internal angles of $122.59^{\circ}$ and $\mathrm{C}-\mathrm{C}$ bond lengths of $d_{C-C}=1.43 \AA$ indicating an elongated chain compared to a similar chain in graphene. The $\mathrm{C}$ atoms are bonded to $\mathrm{N}$ atoms on one side of the interface and $\mathrm{B}$ atoms on the other side, where the N-C bond length is $d_{N-C}=1.40 \AA$, the $\mathrm{C}$ - $\mathrm{B}$ bond is $d_{C-B}=1.53 \AA$. The zigzag-short structure has very simi-

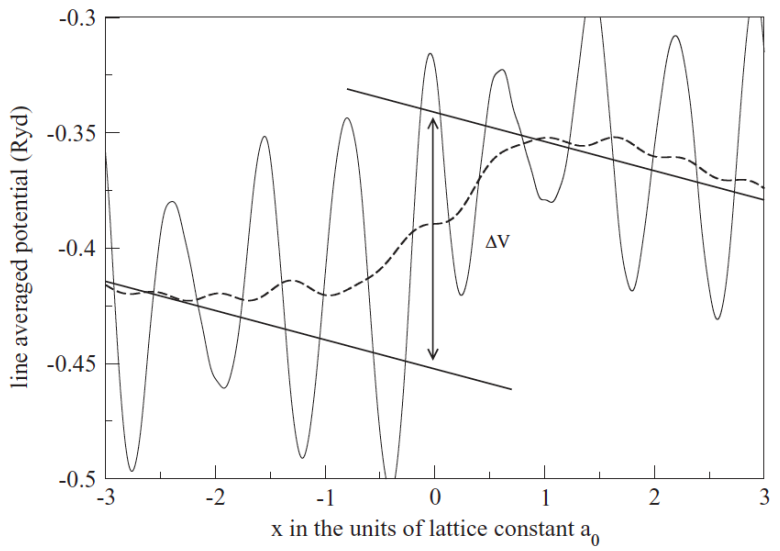

FIG. 1. The BN/C zigzag-short structure showing the band offset with the line-averaged potential shown by the solid line and the macroscopic average the dashed line. Figure is adapted from Ref. 20.

lar internal angles of $119.66^{\circ}$ and bond lengths of $d_{B-N}=$ $1.45 \AA$ at alternating sides to the junction. Both these chains are very similar to those found in pristine boronitrene. The zigzag-short structure has the same characteristics to pristine boronitrene in regions away from the junction.

Using the zigzag-short structure as the reference structure, three different type of strains were applied, namely, (i) biaxial strain with the hexagonal symmetry conserved, (ii) uniaxial strain perpendicular to the interface, and (iii) uniaxial strain parallel to the interface. The compressive strain is studied and presented for completeness. However, unlike tensile strain, compressive strain leads to buckling and instability of the 2D structure. Noteworthy, as mentioned earlier, there are no remnant stresses on the structure.

\section{A. Biaxial strain}

Fig. ?? shows the zigzag-short structure with biaxial strain. The biaxial strain maintains a constant ratio of $\mathrm{L}_{x}$ and $\mathrm{L}_{y}$, therefore conserving hexagonal symmetry. We investigate biaxial strain ranging from -0.1 to 0.25 for the zigzag-short structure. This results in a significant change in the bond length for $d_{N-C}, d_{C-C}$ and $d_{C-B}$ respectively as shown in Fig. 3(a).

There is an increase in the $d_{C-B}$ bond length compared to $d_{C-C}$ and $d_{N-C}$. At about $20 \%$ strain, there is a marked deviation from linearity for $d_{C-B}$. This is attributed to the onset of structural instability leading to distortion in the hexagonal symmetry.

Fig. 3(b) shows the band offset as a function of biaxial strain. The band offset increases under compressive strain and decreases under tensile strain. This provides an avenue for band offset engineering. A band offset discontinuity is observed in the region of the equilibrium 

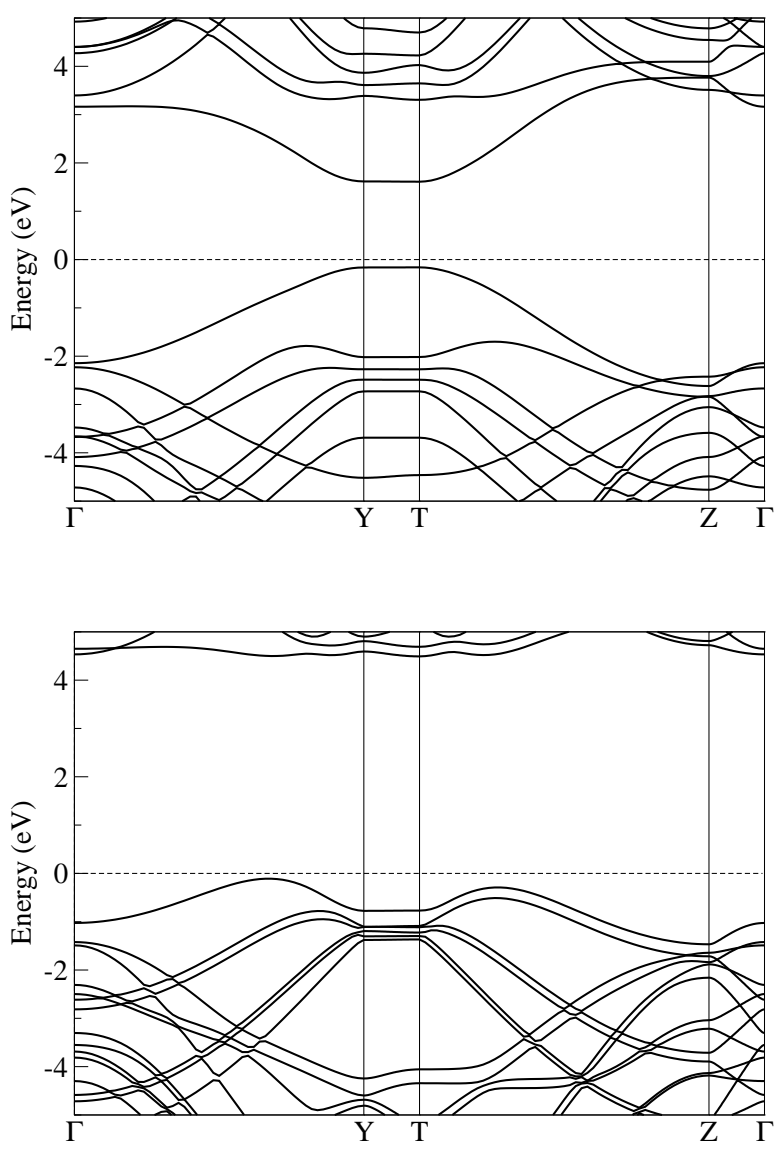

(b) Band Structure of Boronitrene

FIG. 2. Band structure of (a) C-functionalized BN/C (zigzag-short) and (b) boronitrene. $\mathrm{BN} / \mathrm{C}$ zigzag-short is a semi-conductor while boronitrene is a wide band gap semiconductor.

lattice of the ground state structure under biaxial strain. These discontinuities are largely numerical in nature. We conclude that it is difficult to calculate the band offset consistently for very small applied strains which result in small atomic relaxations. These relative errors become less important for larger applied strains. The error bars on the band offsets are of order $\pm 0.02 \mathrm{eV}$ around the region of the equilibrium structure. There is about a $25 \%$ decrease in the band offset in this system for a $15 \%$ application of biaxial strain.

We have demonstrated here that the response to strain of the band offset specifically in this case of BN/C zigzagshort is a different measure to the response to strain of the band gap as reported by Li et al. ${ }^{24}$

\section{B. Uniaxial strain}

Uniaxial strains perpendicular/parallel to the carbon interface were obtained for various values of $\mathrm{L}_{x} / \mathrm{L}_{y}$. Here $\mathrm{L}_{x} / \mathrm{L}_{y}$ is the independent variable and $\mathrm{L}_{y} / \mathrm{L}_{x}$ the dependent variable. The value of $\mathrm{L}_{y} / \mathrm{L}_{x}$ is obtained for a fixed
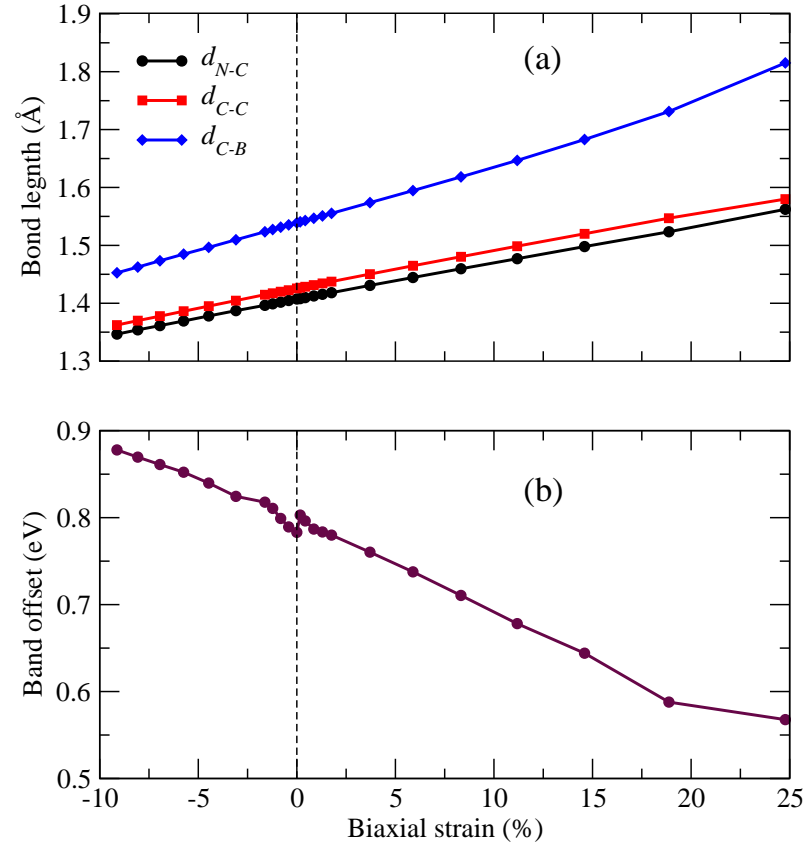

FIG. 3. Consequence of the effect of biaxial strain: (a) on the bond lengths between nitrogen and carbon $\left(d_{N-C}\right)$, carbon and carbon $\left(d_{C-C}\right)$, and carbon and boron $\left(d_{C-B}\right)$. (b) on the band offset. This does not obey the same trend as the band gap and leads to a significant modification of the band offset.

value of $\mathrm{L}_{x} / \mathrm{L}_{y}$ by relaxing the structure in the $y / x$ direction. Uniaxial strains perpendicular/parallel to the carbon interface lead to distortions in the zigzag-short structure. The positive strains and negative strains are tensile and compressive respectively. In Fig. 4(a), the change in $\mathrm{L}_{y}$ as a function of $\mathrm{L}_{x}$ is plotted.

We considered strains ranging between $\pm 4.5 \%$ as shown in Fig .5. There is a significant change in the bond length for $d_{N-C}$ and $d_{C-B}$ compared to $d_{C-C}$ due to the direction of the applied perpendicular strain as shown in Fig. 5a and 5(c). The uniaxial strain perpendicular to the carbon interface results in a slight concave curvature for $d_{N-C}$ and $d_{C-B}$ as a function of applied strain, while $d_{C-C}$ is almost linear except for regions with high tensile strains. For large strains $>5 \%$ the possibilities of anharmonicity and structural instabilities exists, which could lead to significant distortion of the lattice or eventually may lead to the breaking of bonds. Therefore, the band offset obtained within that regime is unrealistic and is hence not reported here. The uniaxial strain parallel to the carbon interface leads to a significant change in the bond lengths $d_{N-C}$ and $d_{C-B}$ compared to $d_{C-C}$ as shown in Fig. 5(c).

In Fig. 5(b) and (d), we observe that the band offset under uniaxial perpendicular/parallel strain around the equilibrium structure has a discontinuity. A similar trend was observed for biaxial strain. We commented earlier that this is due to numerical difficulties in computing the band offset for very small applied strains. The calcu- 


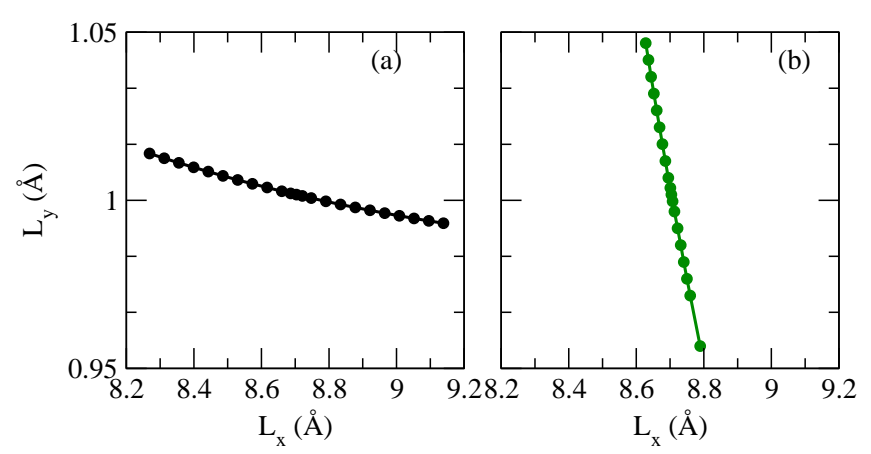

FIG. 4. Anisotropic effect of the two dimensional Cfunctionalized boronitrene termed zigzag short: (a) $L_{y}$ is varied as a function of $L_{x}$ and (b) $L_{x}$ is varied as function $L_{y}$.

lated band offset under uniaxial perpendicular strain for the tensile strain of 0.05 is $0.73 \mathrm{eV}$, and for compressive strain of 0.05 is $0.90 \mathrm{eV}$ (at the equilibrium lattice parameter the band offset is $0.80 \mathrm{eV}$ ). The calculated band offset under uniaxial parallel strain for the tensile strain of $4.5 \%$ is $0.81 \mathrm{eV}$, and at a compressive strain of $-4.5 \%$ is $0.78 \mathrm{eV}$.

\section{ELASTIC PROPERTIES AND PHONON DISPERSION}

In Table I, we present the elastic constants for pristine boronitrene and the zigzag-short structure. The elastic constants for the zigzag-short structure are positive and comparable in value to those obtained for boronitrene.

TABLE I. Elastic constants $c_{i j}$ 's calculated from a leastsquare fit (values in $\mathrm{Nm}^{-1}$ )

\begin{tabular}{rccccc}
\hline \hline & $c_{11}$ & $c_{22}$ & $c_{11 a v e}$ & $c_{12}$ & $c_{66}$ \\
\hline Boronitrene & 289.8 & 289.7 & 289.8 & 63.7 & 113.2 \\
BN/C zigzag-short & 295.5 & 295.6 & 295.6 & 62.01 & 115.2 \\
\hline \hline
\end{tabular}

The following parameters: 2D bulk modulus, 2D shear modulus and 2D young's modulus all have positive values. Therefore, they fulfill the criteria for elastic stability. The Poisson ratio, longitudinal velocity, shear velocity and Debye temperature are presented in Table II for boronitrene and BN/C zigzag-short. The Debye temperature is $2406 \mathrm{~K}$ for boronitrene and $2435 \mathrm{~K}$ for zigzag-short, which is obtained from the mean velocity (resulting from the longitudinal and transverse velocity). This gives an indication of the stiffness of the material. The Debye temperature can also be estimated from the phonon dispersion curve (the maximum frequency multiplied by the Planck's constant divided by Boltzmann constant). This is the temperature were all the phonon modes are excited. The bulk modulus of the zigzag-short is $178 \mathrm{Nm}^{-1}$, which is slightly higher than that of boronitrene $\left(176 \mathrm{Nm}^{-1}\right)$ but well below that of graphene.
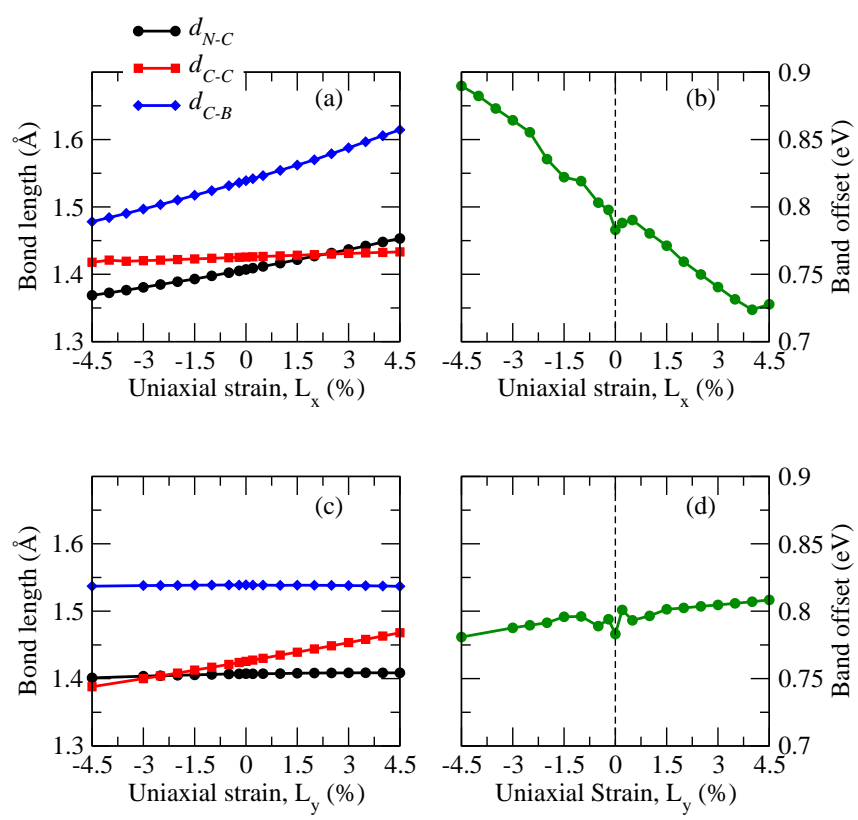

FIG. 5. Uniaxial strain property between $\pm 4.5 \%$; (Top panel) (a) is the effect of bond length as function of unixial strain along the $L_{x}$ and (b) is the corresponding band offset of the uniaxial strain of $L_{x}$. (Bottom panel) (c) is the effect of bond length as strain along $L_{y}$ is varied between $\pm 4.5 \%$ and (d) is the corresponding band offset of the uniaxial strain of $L_{y}$.

Boronitrene and graphene are known to be almost lattice matched. The presence of carbon in boronitrene does not significantly alter the elastic properties when compared to pristine boronitrene. In Table I and II, we observe a slight increase in the elastic properties.

The phonon dispersion for BN/C-zigzag structure is presented in Fig. 6 to affirm the dynamic stability of the BN/C structure. The phonon dispersion calculated exploits the linearity relationship between the induced forces on the atoms in the crystal and the displacement of the atoms from their equilibrium position, which holds within the harmonic regime.

In Fig. 6, no negative phonon modes are observed in the phonon dispersion plot, hence this structure is dynamically stable. The phonon modes are evenly distributed on the dispersion plot due to the relatively similar masses of the atoms in the compound. Owing to the fact that graphene and boronitrene is almost latticed matched, the lattice is not perturbed significantly. Hence, the phonon frequency affirms our assertion that the system is stable.

\section{CONCLUSIONS}

This study is motivated by the usefulness of allotropes of boron nitride, the possibility of the creation of $2 \mathrm{D}$ nanoscale devices and the similarities of its twodimensional structures to graphene. We modified the 
TABLE II. Calculated values for various parameters: bulk modulus $\left(B^{2 D}\right)$, shear modulus $\left(G^{2 D}\right)$, Young's modulus $\left(Y^{2 D}\right)$, Poisson ratio, transverse velocity, longitudinal velocity, mean velocity, Debye temperature. Moduli in $\mathrm{Nm}^{-1}$, acoustic velocities in $\mathrm{ms}^{-1}$, Debye temperature in Kelvin.

\begin{tabular}{rccccccc}
\hline \hline & $B^{2 D}$ & $G^{2 D}$ & $Y^{2 D}$ & $\nu$ & $\mathrm{v}_{t}$ & $\mathrm{v}_{l}$ & $\mathrm{v}_{m}$ \\
\hline Boronitrene & 176.7 & 113.2 & 275.8 & 0.22 & 12252 & 19607 & 14694 \\
BN/C zigzag-short & 178.8 & 115.2 & 282.5 & 0.21 & 12382 & 19835 & 14854 \\
\hline \hline
\end{tabular}

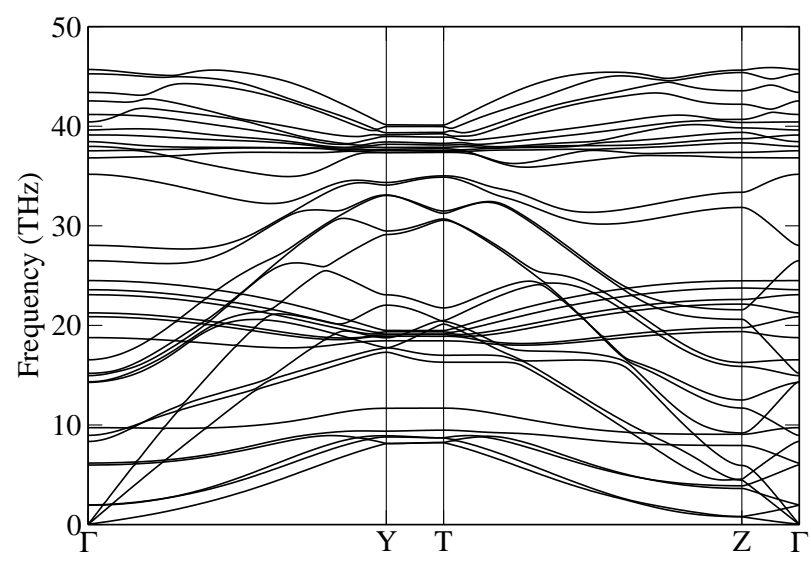

FIG. 6. Phonon dispersion of zigzag-short C-functionalized $\mathrm{BN}$.

band gap of boronitrene by the introduction of a double line of carbon atoms in pristine boronitrene in the so-called "zigzag-short" structure. In order to study the effect of strain on the band offset, three different strains were applied to this structure. The strains result in a substantial change of the band offset for this system therefore giving an ideal route to engineer the band offset in this system.

We compared the elastic properties of BN/C zigzagshort in relation to pristine boronitrene. The elastic properties of pristine broronitrene and $\mathrm{BN} / \mathrm{C}$ zigzagshort show no marked difference. The calculated elastic constants for the BN/C zigzag-short structure show that this system is elastically stable at $\mathrm{T}=0 \mathrm{~K}$ and zero pressure as indicated by their positive values. The band structure shows that BN/C zigzag-short structure undergoes modification in its band gap compared to pristine boronitrene. Because of the reduced band gap, BN/C zigzag-short is useful in areas where high elastic and mechanical strength is paramount but a reduced band gap is essential.

\section{ACKNOWLEDGMENTS}

We are grateful to the National Research Foundation through the University of Pretoria and University of South Africa for financial support. We thank the center for high performance computing (CHPC) South Africa for use of their computational resources. KOO is grateful to Prof Moritz Braun for support.
* obodoko@unisa.ac.za

† joshua.obodo@unn.edu.ng

1 P. Rani and V. K. Jindal, RSC Advances 3, 802 (2013).

2 M. Molepo, R. Mapasha, K. Obodo, and N. Chetty, Computational Materials Science 92, 395 (2014).

3 R. Drost, A. Uppstu, F. Schulz, S. Hmlinen, M. Ervasti, A. Harju, and P. Liljeroth, Nano Letters 14, 5128 (2014).

${ }^{4}$ L. Liu, J. Park, D. A. Siegel, K. F. McCarty, K. W. Clark, W. Deng, L. Basile, J. C. Idrobo, A.-P. Li, and G. Gu, Science 343, 163 (2014).

5 A. M. Ukpong, Computational Condensed Matter 2, 1 (2015)

${ }^{6}$ R. Drost, S. Kezilebieke, M. Ervasti, S. Hmlinen, F. Schulz, A. Harju, and P. Liljeroth, Scientific Reports 5 (2015), $10.1038 /$ srep16741.

7 X. Wei, S. Xiao, F. Li, D.-m. Tang, Q. Chen, and Y. Bando, Nano Letters 15, 689 (2015).

8 J. T. Obodo, K. O. Obodo, and U. Schwingenschlögl, New Journal of Physics 17, 93012 (2015).

9 R.-N. Wang, M. Yang, G.-Y. Dong, and S.-F. Wang, Journal of Physics: Condensed Matter 28, 55302 (2016).
10 J. C. Dong and H. Li, The Journal of Physical Chemistry C 116, 17259 (2012).

11 C.-K. Chang, S. Kataria, C.-C. Kuo, A. Ganguly, B.Y. Wang, J.-Y. Hwang, K.-J. Huang, W.-H. Yang, S.-B. Wang, C.-H. Chuang, M. Chen, C.-I. Huang, W.-F. Pong, K.-J. Song, S.-J. Chang, J.-H. Guo, Y. Tai, M. Tsujimoto, S. Isoda, C.-W. Chen, L.-C. Chen, and K.-H. Chen, ACS Nano 7, 1333 (2013).

12 Z. Liu, X. Zhong, H. Yan, and R.-Z. Wang, Physical Chemistry Chemical Physics 18, 974 (2016).

13 K. S. Novoselov, a K Geim, S. V. Morozov, D. Jiang, Y. Zhang, S. V. Dubonos, I. V. Grigorieva, and A. A. Firsov, Science 306, 666 (2004).

${ }^{14}$ K. S. Novoselov, D. Jiang, F. Schedin, T. J. Booth, V. V. Khotkevich, S. V. Morozov, and A. K. Geim, PNAS 102, 10451 (2005).

15 H. Sachdev, F. Müller, and S. Hüfner, Diamond \& Related Materials 19, 1027 (2010).

${ }^{16}$ Li Song, Lijie Ci, Hao Lu, Pavel B Sorokin, Chuanhong Jin, Jie Ni, Alexander G Kvashnin, Dmitry G Kvashnin, Jun Lou, Boris I Yakobson, and Pulickel M Ajayan, Nano 
Letters 10, 3209 (2010).

17 M. Topsakal, E. Aktürk, and S. Ciraci, Physical Review B 79, 115442 (2009).

18 H. Şahin, S. Cahangirov, M. Topsakal, E. Bekaroglu, E. Akturk, R. T. Senger, and S. Ciraci, Physical Review B 80, 155453 (2009).

19 T. B. Ngwenya, A. M. Ukpong, and N. Chetty, Physical Review B 84, 1 (2011).

${ }^{20}$ K. O. Obodo, R. C. Andrew, and N. Chetty, Physical Review B 84, 155308 (2011).

21 S. W. King, M. French, J. Bielefeld, M. Jaehnig, M. Kuhn, G. Xu, and B. French, Applied Physics Letters 101 (2012).

22 Y. Lin and J. W. Connell, Nanoscale 4, 6908 (2012).

23 J. Kang, S. Tongay, J. Zhou, J. Li, and J. Wu, Applied Physics Letters 102, 012111 (2013).

24 J. Li, G. Gui, and J. Zhong, Journal of Applied Physics 104, 094311 (2008).

${ }^{25}$ R. C. Andrew, R. E. Mapasha, a. M. Ukpong, and N. Chetty, Physical Review B 85, 125428 (2012).
${ }^{26}$ P. Hohenberg and W. Kohn, Physical Review 136, 864 (1964).

27 G. Kresse and J. Furthmüller, Physical review B 54, 11169 (1996).

28 P. E. Blöchl, Physical Review B 50, 17953 (1994).

29 John P Perdew, Kieron Burke, and Matthias Ernzerhof, Physical Review Letters 77, 3865 (1996).

30 Hendrik J Monkhorst and James D Pack, Physical Review B 13, 5188 (1976)

31 M. Methfessel and A. T. Paxton, Physical Review B 40, 3616 (1989).

32 Peter E Blochl, O. Jepsen, and O. K. Andersen, Physical Review B 49, 16223 (1994).

33 Yvon Le Page and Paul Saxe, Physical Review B 65, 104104 (2002).

34 A. Baldereschi, S. Baroni, and R. Resta, Physical Review Letters 61, 734 (1988).

35 K. Parlinski, Z. Q. Li, and Y. Kawazoe Phys. Rev. Lett. 78, 4063 (1997) 